ALICE-PUB-2001-10

9 April 2001

\title{
The STAR-RICH Detector
}

B. Lasiuk ${ }^{\mathrm{a}}$, for the STAR-RICH Collaboration (Bari ${ }^{1} / \mathrm{CERN}^{2}$ ALICE-HMPID, Yale ${ }^{3}$ ) and the STAR* Collaboration.

The STAR-RICH Collaboration: Y. Andres ${ }^{2}$, A. Braem ${ }^{2}$, D. Cozza ${ }^{1}$, M. Davenport ${ }^{2}$, G. De Cataldo ${ }^{1}$, L. Dell Olio ${ }^{1}$, D. DiBari ${ }^{1}$, A. DiMauro ${ }^{2}$, J. C. Dunlop ${ }^{3}$, E. Finch ${ }^{3}$, D. Fraissard ${ }^{2}$, A. Franco ${ }^{1}$, J. Gans ${ }^{3}$, B. Ghidini ${ }^{1}$, J. W. Harris ${ }^{3}$, M. Horsley ${ }^{3}$, G. J. Kunde ${ }^{3}$, B. Lasiuk ${ }^{3}$, Y. Lesenechal², R. D. Majka ${ }^{3}$, P. Martinengo ${ }^{2}$, A. Morsch², E. Nappi ${ }^{1}$, G. Paic ${ }^{2}$, F. Piuz ${ }^{2}$, F. Posa ${ }^{1}$, J. Raynaud ${ }^{2}$, S. Salur ${ }^{3}$, J. Sandweiss ${ }^{3}$, J. C. Santiard ${ }^{2}$, J. Satinover ${ }^{3}$, E. Schyns ${ }^{2}$, N. Smirnov ${ }^{3}$, J. Van Beelen ${ }^{2}$, T. D. Williams², Z. $\mathrm{Xu}^{3}$

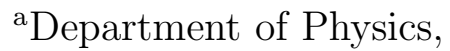
Yale University,

New Haven CT, 06511

The STAR-RICH detector extends the particle identification capabilities of the STAR spectrometer for charged hadrons at mid-rapidity. It allows identification of pions and kaons up to $\sim 3 \mathrm{GeV} / \mathrm{c}$ and protons up to $\sim 5 \mathrm{GeV} / \mathrm{c}$. The characteristics and performance of the device in the inaugural RHIC run are described.

\section{Introduction}

Interactions which occur at large momentum transfer $\left(\mathrm{Q}^{2}\right)$ are well described by perturbative QCD (pQCD) calculations. These hard processes produce particles with high transverse momentum $\left(\mathrm{p}_{T}\right)$. Such processes have never occurred at significant rates in nucleus-nucleus collisions before the advent of RHIC. These penetrating probes are believed to be sensitive to the state of hot dense nuclear matter that is produced in these collisions and their spectral yields are affected by how the excited medium affects propagating partons. Flavor dependence of particle production at high $\mathrm{p}_{T}$ is predicted to be sensitive to differences in the properties of quark and gluon jets in dense matter [1]. This provides the motivation for particle identification at high momentum.

The main Particle IDentification (PID) capabilities of STAR rest on the ionization measurements in the tracking detectors. In the relativistic rise the resolution of these measurements is not sufficient to discriminate between particle species. In order to extend the PID capabilities of STAR, a Ring Imaging CHerenkov (RICH) detector has been installed covering a region $|\eta|<.30$ and $\Delta \phi=20^{\circ}$.

The CERN RD-26 collaboration demonstrated that thin-film CsI is a viable alternative to TMAE photo converters for RICH detectors [2] and this allowed the ALICE collaboration to develop a $1 \mathrm{~m}^{2}$ prototype chamber. This formed the basis for the HMPID detector

\footnotetext{
${ }^{*}$ For complete author list see J.W. Harris, these proceedings.
} 
proposal for the ALICE experiment at LHC [3]. The ALICE-LHC and STAR-RHIC environments are similar in terms of detector topology both having a large TPC in the central region. As such, the development of a RICH detector for the ALICE experiment was directly applicable to STAR. It was this module which was adapted to fit into the constraints and infrastructure of the STAR experiment [4]. It was installed for the first three years at RHIC by a joint Yale-Bari-CERN collaboration.

\section{Characteristics}

The reconstruction of the Cherenkov angle requires two-dimensional positional information of the Cherenkov photons with respect to the incident track. To do this over an area of $\sim 1 \mathrm{~m}^{2}$, a thin $(0.3 \mu \mathrm{m})$ CsI film was deposited on a segmented cathode pad plane possessing 15,360 pads of dimension $8.0 \times 8.4 \mathrm{~mm}^{2}$. This provides a means for photoconversion of the Cherenkov photons. In conjunction with a Multi-Wire Proportional Chamber (MWPC), both photo-electron and charged particle 2-D localization is possible.
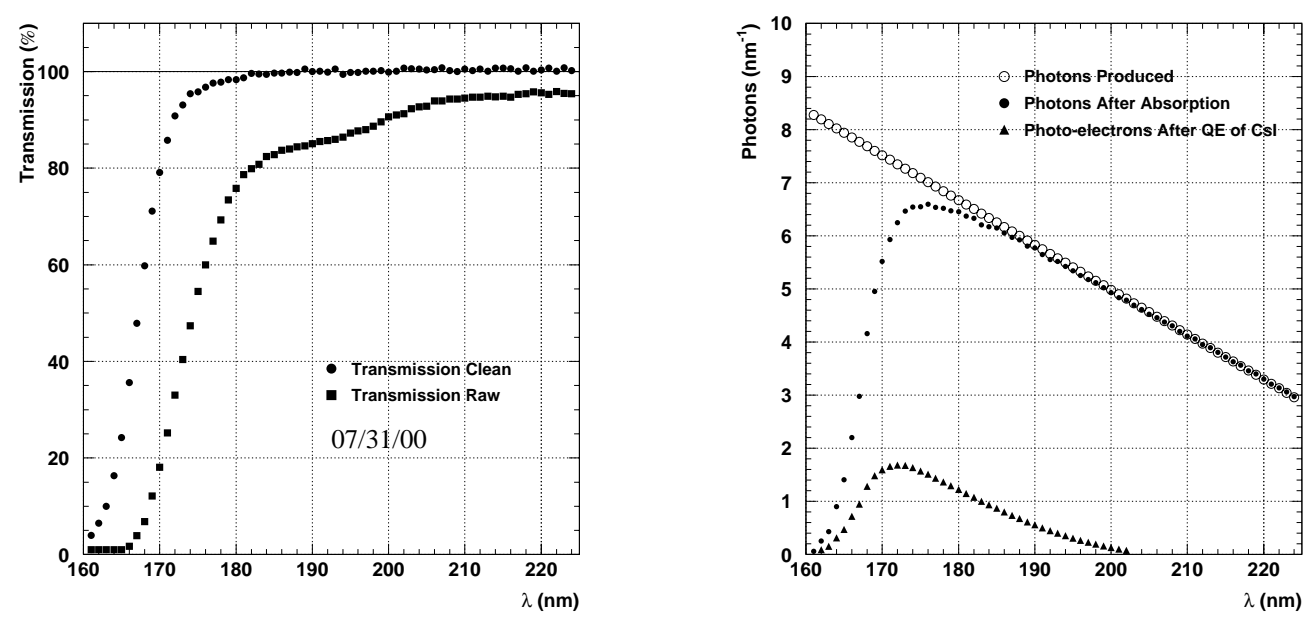

Figure 1. The left panel shows the measured transmission of the liquid radiator in raw (squares) and dehydrated (circles) form. The right panel shows the simulated spectrum of Cherenkov light produced in the liquid radiator (open circles) and the effect of the finite liquid and quartz transmission. The resulting spectrum transmitted to the pad plane (solid circles) is folded with the quantum efficiency of the CsI to show the spectrum of photons converted to electrons (triangles).

The CsI photo-cathode provides a planar surface for the photo-conversion and emission to occur. However, CsI has a photo-electric threshold of $6.0 \mathrm{eV}$ which restricts its sensitivity to vacuum ultra-violet (VUV) photons. This imposes constraints on the spectral properties of the radiator medium, its containment vessel, as well as the MWPC gas. In order to extend the PID capabilities of the TPC, a radiator with an index of refraction in the range of 1.2-1.3 is necessary. Perfluorohexane $\left(\mathrm{C}_{6} \mathrm{~F}_{14}\right)$ has an index of refraction of $\sim 1.285$ at $170 \mathrm{~nm}$ [3], and shows transparency to VUV radiation down to $152 \mathrm{~nm}$, if water and oxygen are reduced to the ppm level. A $1 \mathrm{~cm}$ thick layer of liquid is confined 
by a $0.5 \mathrm{~cm}$ thick quartz windowed vessel in order to transmit the Cherenkov photons generated in the liquid. Pure methane is utilized as the MWPC gas since it is transparent to VUV radiation down to $\sim 130 \mathrm{~nm}$ and allows the chamber to be run in a stable manner at high gas gain. The importance of a transparent radiator medium is depicted in figure 1. The left panel shows the measured transmission of the liquid before and after dehydration while the right panel shows the simulated spectrum of Cherenkov light which is incident on the pad plane and subsequently converted to photo-electrons.

In addition to the spectral constraints CsI imposes, it is also hygroscopic and care must be taken to isolate it from water, as well as oxygen. Water at the 10-100s of ppm level may degrade the quantum efficiency of the CsI layer. As such, a continuous anhydrous gas flow must be present to buffer the CsI from contaminants and out-gassing products from within the detector. This was a significant consideration in transporting the detector from CERN to BNL. A series of tests were done at a CERN test beam which exposed the photo-cathodes to a controlled amount of water and oxygen and the quantum efficiency measured before and after the test. This provided constraints for construction of a sealed transportation vessel [5].

\section{Performance and Analysis}

In the first year of running, STAR recorded 900k central Au-Au and 900k minimum bias $\mathrm{Au}-\mathrm{Au}$ triggers at $\sqrt{s_{N N}}=130 \mathrm{GeV} / \mathrm{A}$. For the central $\mathrm{Au}-\mathrm{Au}$ collisions, the RICH occupancy never exceeded $5 \%$ of the total number of pads. For these events, an average of 1 track with a momentum more than $1 \mathrm{GeV} / \mathrm{c}$ pass through the detector. An example of an off-line event display is shown in figure 2 . The gain of the chamber was set at $\sim 2 \times 10^{5}$ in order to keep the number of saturated pads to a minimum while keeping a single electron detection efficiency above $80 \%$. The average incident angle for tracks which impinge on the RICH are larger at STAR than expected at ALICE because of the distance from the interaction region and the magnitude of the magnetic field. This produces Cherenkov "rings" which are elliptical in shape.

The identification of the charged particles in the RICH requires tracks from the TPC to be extrapolated and associated with an ionization hit in the RICH. The efficiency of this matching is dependent on the precision of the extrapolation as well as the capability to distinguish between a hit from a photon and charged particle track. This discrimination is done by threshold cuts on the quantity of charge contained within a hit. Extrapolation of tracks to the RICH show a residual distribution with a $\sigma$ of $\sim 4.2 \mathrm{~mm}$ for tracks with a momentum greater than $1 \mathrm{GeV} / \mathrm{c}$. Tracks with smaller momentum have a much larger width since multiple scattering becomes more appreciable.

Once the association is done, two identification algorithms are used. The first method utilizes a Hough transformation which converts the photon coordinates relative to the track impact point into a Cherenkov angle [6]. This angle is used to reconstruct the mass of the particle in association with the momentum given by the TPC. The second method makes three mass hypotheses for a given track $(\pi, \mathrm{K}$, and $\mathrm{p})$, and the limiting bounds of the Cherenkov rings, defined by the dimensions of the radiator vessel, are projected on to the pad plane. These bands are illustrated in figure 2. The mass of the particle can be assigned by selecting the band with the highest density of photons. Using these two 
algorithms, values for the ratio of the anti-proton to proton yield in central $130 \mathrm{GeV} / \mathrm{A}$ $\mathrm{Au}-\mathrm{Au}$ collisions were extracted. They are reported elsewhere in this volume [7].

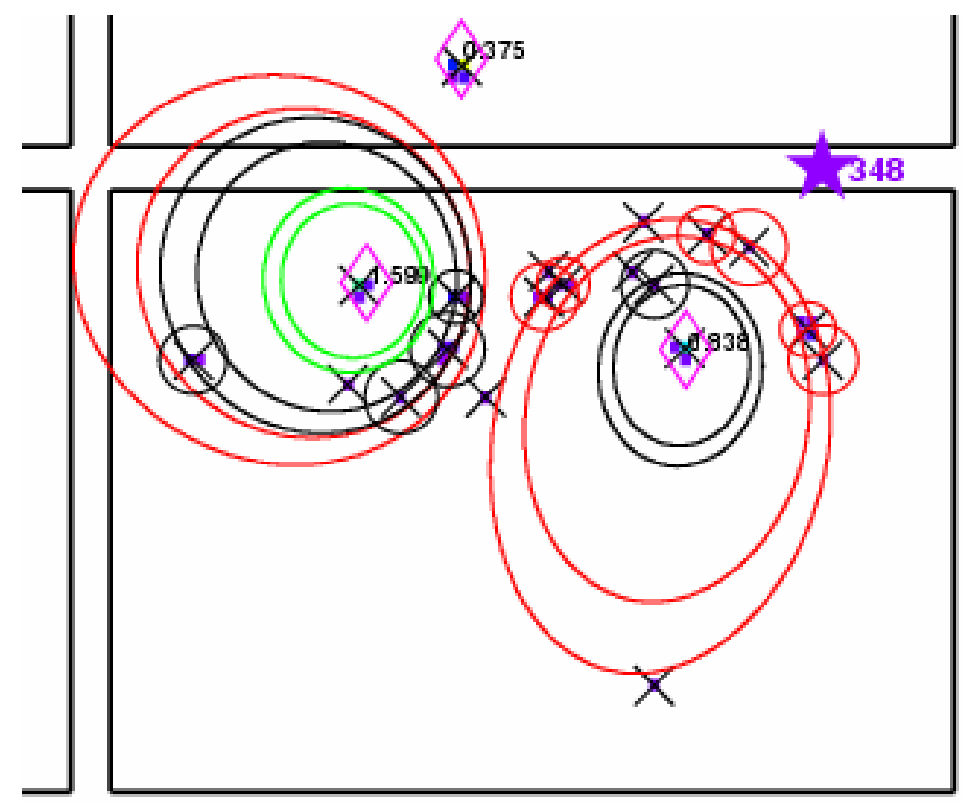

Figure 2. An off-line event display of 1 quadrant of the RICH detector showing an identified $\pi$ and $\mathrm{K}$ in a semi-central event. The x's mark the position of a reconstructed hit while the diamonds indicate the position of an extrapolated track from the TPC. The rings show the regions where photons are expected for different mass hypotheses.

\section{Conclusion}

A RICH detector, developed by the ALICE collaboration has been installed in the STAR detector at RHIC. This device extends the PID capabilities of the spectrometer to $\sim 3 \mathrm{GeV} / \mathrm{c}$ for pions and kaons, and $\sim 5 \mathrm{GeV} / \mathrm{c}$ for protons. This detector has produced an anti-proton to proton ratio in the transverse momentum range $2.0<\mathrm{p}_{T}<2.5(\mathrm{GeV} / \mathrm{c})$ at mid-rapidity for $130 \mathrm{GeV} / \mathrm{A}$ Au-Au collisions. This measurement will be extended with further data in the coming year which will allow an examination of the effect of high density matter on the fragmentation distribution of high $\mathrm{p}_{T}$ partons.

\section{REFERENCES}

1. for example see X. N Wang, Phys. Rev C58 (1998) 2231.

2. E. Nappi et al., CERN/DRDC 92-3, proposal P35.

3. ALICE collaboration, ALICE Technical Design Report: Detector for High Momentum PID, CERN/LHCC 98-19.

4. STAR-RICH collaboration, Proposal for a Ring Imaging Cherenkov Detector in STAR, YRHI 98-022.

5. Di Mauro et al., NIM A461 (2001) 584.

6. D. Elia et al., NIM. A433 (1999) 262.

7. J. C. Dunlop for the STAR collaboration, these proceedings. 